\title{
Expression of DACT1 in children with asthma and its regulation mechanism
}

\author{
CUNXUE ZHANG ${ }^{1}$, PEILI YANG ${ }^{1}$, YAN CHEN $^{1}$, JING LIU $^{2}$ and XIUTAI YUAN ${ }^{1}$ \\ ${ }^{1}$ Department of Pediatrics, The First People's Hospital of Jining, Jining, Shandong 272000; ${ }^{2}$ Institute of \\ Stem Cell and Regenerative Medicine, Medical College, Xiamen University, Xiamen, Fujian 361102, P.R. China
}

Received June 15, 2016; Accepted April 28, 2017

DOI: 10.3892/etm.2018.5706

\begin{abstract}
The aim of the present study was to detect DACT1 expression levels in the lungs of children with asthma, and to investigate its role and molecular mechanisms in regulating the expression of inflammatory factors in RAW264.7 cells. DACT1, DACT2 and DACT3 expression was analyzed in biopsy specimens from 10 cases of newly diagnosed children with asthma and 10 healthy controls by reverse transcription-quantitative polymerase chain reaction, and their expression was confirmed in RAW264.7 cells. DACT1 expression was silenced by small interfering RNA or enhanced by transfection of pcDNA-3.1-DACT1 in RAW264.7 cells, and expression of $\beta$-catenin and inflammatory factors, interleukin (IL) 5, IL6 and IL13, was analyzed. Nuclear translocation of $\beta$-catenin was detected by western blot analysis, and the effect of DACT1 on $\beta$-catenin was investigated with rescue experiments. Regulation of the Wnt signaling pathway by DACT1 and $\beta$-catenin was analyzed in RAW264.7 cells after recombinant Wnt5A stimulation. DACT1, DACT2 and DACT3 were significantly upregulated in specimens from children with asthma compared with controls $(\mathrm{P}<0.05)$ and the expression of DACT1 was significantly more increased compared with DACT2 and DACT3 $(\mathrm{P}<0.05)$. Inhibition of DACT1 expression significantly suppressed IL5, IL6 and IL13 mRNA expression levels compared with the control $(\mathrm{P}<0.05)$, while upregulated DACT1 expression significantly increased IL5, IL6 and IL13 mRNA expression $(\mathrm{P}<0.05)$. DACT1 inhibited the expression and nuclear translocation of $\beta$-catenin, while overexpression of $\beta$-catenin significantly inhibited the biological function of DACT1 $(\mathrm{P}<0.05)$. Overexpression of $\beta$-catenin also significantly suppressed the upregulation of IL5, IL6 and IL13 mRNA induced by pcDNA3.1-DACT1 transfection $(\mathrm{P}<0.05)$. Following the addition of Wnt5A, overexpression of DACT1 inhibited the expression and nuclear translocation of $\beta$-catenin, and upregulated IL5, IL6 and IL13 mRNA expression. In conclusion,
\end{abstract}

Correspondence to: Professor Peili Yang, Department of Pediatrics, The First People's Hospital of Jining, 6 Jiankang Road, Jining, Shandong 272000, P.R. China

E-mail: ypeili01@163.com

Key words: DACT, $\beta$-catenin, child asthma
DACT1 was indicated to be upregulated in lung tissues from children with asthma, which could induce higher pro-inflammatory factor expression. DACT1 may act via inhibiting the expression and nuclear translocation of $\beta$-catenin, a factor in the Wnt signaling pathway. The present results suggested that DACT1 may be a potential target for the treatment of asthma.

\section{Introduction}

Bronchial asthma (asthma) is an airway chronic persistent inflammatory disease in the respiratory tract. It is accompanied by increased airway reactivity that involves a variety of inflammatory cells (including $\mathrm{T}$ lymphocytes, eosinophils, neutrophils and mast cells), structural cells (including smooth muscle cells and respiratory epithelial cells) and other cell types, and acute attacks of this disease can endanger the patient's life (1-3). A recent study indicated that the incidence of asthma in children increases year by year, which has attracted the attention of researchers worldwide (4). Currently, long-term inhaled corticosteroid treatment is the principal method of treating asthma clinically, which alleviates the symptoms of the condition. However, hormone resistance or dependence, and drug-induced side effects of hormones, such as femoral head necrosis and growth suppression, may occur simultaneously $(5,6)$. Asthma is primarily characterized by chronic airway inflammation, airway hyperresponsiveness and airway irreversible remodeling $(7,8)$, among which airway remodeling is directly related to the prognosis of patients with asthma. Recent studies confirmed the involvement of inflammatory, smooth muscle and epithelial cells in this process; however, the molecular mechanism involved remains unclear $(9,10)$.

DACT1 is a member of the Dapper family, which includes three genes: DACT1, DACT2 and DACT3 (11). As a tumor suppressor, DACT1 is downregulated in multiple tumor types, such as liver, colon and breast cancer (12). It has been demonstrated that DACT1 functions in the development of tumors primarily by regulating signaling pathways, such as Wnt and the c-Jun N-terminal kinase pathway (13-15). For instance, DACT1 binds to disheveled homolog 1 (Dvl1) to induce the degradation of Dvl1 and the subsequent inhibition of the Wnt/ $\beta$-catenin pathway (16). DACT1 may also bind to Dvl2, affecting cell movement ability by regulating cell polarity (17). The Wnt/ $\beta$-catenin signaling pathway is involved in the regulation of numerous physiological and pathological processes, and 
is closely associated with multiple diseases. Previous studies have indicated that the $\mathrm{Wnt} / \beta$-catenin pathway is associated with lung development, pulmonary fibrosis, airway remodeling and inflammation $(18,19)$. For example, TGF- $\beta 1$ stimulation of human lung fibroblast cells upregulates Wnt pathway-related gene expression, such as the expression of Wnt5b, frizzled-8 and Dvl3, and induces differentiation of fibroblasts into myofibroblasts, and these effects could be alleviated by inhibition of the Wnt signaling pathway (20). In addition, the Wnt signaling pathway may inhibit IL6 expression in asthma effector cells, thereby reducing the local inflammatory response (21). Based on these findings, it was proposed that DACT1 may be involved in asthma airway inflammation via regulation of the Wnt signaling pathway. In the present study, the macrophage cell line, RAW264.7, was used to investigate the regulatory mechanisms and biological effects of DACT1 on the Wnt signaling pathway.

\section{Materials and methods}

Patients. A total of 10 child patients (mean age, 12 years; 3 males and 7 females) with asthma that were first diagnosed by electronic bronchoscope and 10 healthy controls were recruited from the First People's Hospital of Jining (Jining, China) between November 2013 and January 2015. The patients were admitted to the Department of Pediatrics, The First People's Hospital of Jining (Jining, China) and the healthy controls (mean age, $15.5 \pm 1.0$ years; 6 males and 4 females) were recruited at the Physical Examination Center of the First People's Hospital of Jining. In these controls, there was no history of chronic diseases, organic disease, no history of prolonged medication use and no acute diseases were indicated during physical examination. Patients with asthma were children who were first diagnosed with mild or medium asthma, and none of the subjects accepted hormone treatment in the 4 weeks prior to diagnosis. Lung tissues from patients (pathological tissues) and healthy controls (normal tissues) were collected and stored in $-80^{\circ} \mathrm{C}$ following freezing by liquid nitrogen. Prior to collection of tissues, written informed consent was obtained from the patients' families and the study was approved by the Ethics Review Board of the First People's Hospital of Jining.

Cell culture and transfection. Mouse RAW264.7 cells were purchased from the Cell Center of Chinese Academy of Science (Shanghai, China) and cultured in high glucose Dulbecco's modified Eagle's medium (DMEM; Gibco; Thermo Fisher Scientific, Inc., Waltham, MA, USA) supplemented with $10 \%$ heat-inactivated fetal bovine serum (FBS; Gibco; Thermo Fisher Scientific, Inc., Waltham, MA, USA) and $1 \%$ penicillin-streptomycin. Cultures were incubated in a humidified atmosphere with $5 \% \mathrm{CO}_{2}$ at $37^{\circ} \mathrm{C}$ and passaged every 24-48 h when confluence reached $90 \%$.

A total of $2 \times 10^{5}$ RAW264.7 cells were inoculated into a 24-well plate with DMEM supplemented with $10 \%$ FBS without antibiotics at $37^{\circ} \mathrm{C}$. When the confluence reached $70 \%$, cells were used for transfection. A total of $2 \times 10^{5}$ cells were transfected with 25 pmol of mature DACT1 small interfering (si)RNA (siR-DACT1; Hanheng Biotechnology Co., Ltd., Shanghai, China) (forward, 5'-AGUUCAAUUGCU UUCUUAGCA-3' and reverse, 5'-CUAAGAAAGCAAUUG
Table I. Primers used for quantitative polymerase chain reaction.

\begin{tabular}{ll} 
Primers & \multicolumn{1}{c}{ Primer sequence } \\
\hline DACT1_Forward & 5'-AGATATCCCCTTGGCACCCT-3' \\
DACT1_Reverse & 5'-TTCAGTGAGAGTCCACCACA-3' \\
DACT2_Forward & 5'-GGCCCCCTCTCATTCACAAA-3' \\
DACT2_Reverse & 5'-CATTCAGTGAGAGTCCACCACA-3' \\
DACT3_Forward & 5'-AGGGTCAAGTAGGGGTTGGT-3' \\
DACT3_Reverse & 5'-ATTCAGTGAGAGTCCACCACA-3' \\
3-catenin_Forward & 5'-TGGAACATGAGATGGGTGGC-3' \\
3-catenin_Reverse & 5'-GTGTTCTACACCATTACTCAATTCT-3' \\
IL13_Forward & 5'-GCAATGGCAGCATGGTATGG-3' \\
IL13_Reverse & 5'-CCCGCCTACCCAAGACATTT-3' \\
IL5_Forward & 5'-AACTGTGCAAGGGGGTACTG-3' \\
IL5_Reverse & 5'-AGGCCTGACTCTTTCTTGGC-3' \\
IL6_Forward & 5'-TCAATATTAGAGTCTCAACCCCCA-3' \\
IL6_Reverse & 5'-TTCTCTTTCGTTCCCGGTGG-3' \\
GAPDH_Forward & 5'-ACCACAGTCCATGCCATCAC-3' \\
GAPDH_Reverse & 5'-TCCACCACCCTGTTGCTGTA-3'
\end{tabular}

IL, interleukin.

AACUGU-3') using Lipofectamine 2000 (Thermo Fisher Scientific, Inc.), according to the manufacturer's instructions. As a control, RAW264.7 cells $\left(2 \times 10^{5}\right)$ were transfected with negative control siRNA (Hanheng Biotechnology Co., Ltd.) without specifically targeting any human gene products. For overexpression of the DACT1 gene, cells were transfected with $300 \mu \mathrm{g}$ pcDNA3.1-DACT1, pcDNA3.1- $\beta$-catenin or an empty vector (Hanheng Biotechnology Co., Ltd.) using Lipofectamine 2000. Cells were collected at 48 h post-transfection for further experiments. Cells transfected with siRNA negative control were considered the NC group and cells transfected with pcDNA3.1 empty vector were considered the empty vector group.

RNA extraction and reverse transcription-quantitative polymerase chain reaction ( $R T-q P C R)$ assay. Total RNA was extracted from cells and tissues using TRIzol reagent (Invitrogen; Thermo Fisher Scientific, Inc.), according to the manufacturer's instructions. A total of $1 \mathrm{ml}$ TRIzol reagent was added to $100 \mathrm{mg}$ tissue or a single cell layer in a $10-\mathrm{cm}$ culture plate. RNA was reverse transcribed into cDNA using a Takara PrimeScript RT Reagent kit (Takara Biotechnology Co., Ltd., Dalian, China), according to the manufacturer's instructions. Subsequently, qPCR was performed using a SYBR PrimeScript RT-PCR kit (Takara Biotechnology Co., Ltd.), according to the manufacturer's instructions. The expression of GAPDH was used as an internal control for mRNA. The reaction mixture was incubated for 1 cycle at $95^{\circ} \mathrm{C}$ for $10 \mathrm{~min}$, followed by 40 cycles at $95^{\circ} \mathrm{C}$ for $30 \mathrm{sec}$ and $60^{\circ} \mathrm{C}$ for $30 \mathrm{sec}$. Primers used for amplification are demonstrated in Table I. The relative expression levels were evaluated using the $2^{-\Delta \Delta C q}$ method (22) and repeated for three times. 
A



B

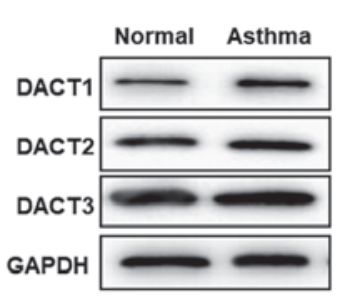

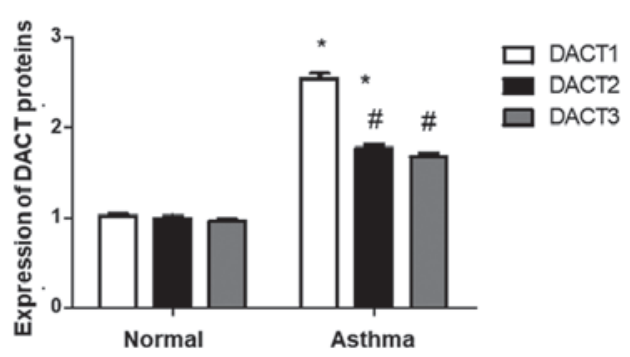

Figure 1. Expression of DACT1, DACT2 and DACT3 is upregulated in children with asthma. Lung tissues were collected from 10 cases of newly diagnosed children with asthma and 10 healthy controls. (A) Expression of DACT1, DACT2 and DACT3 mRNA was analyzed by reverse transcription-quantitative polymerase chain reaction. (B) DACT1, DACT2 and DACT3 protein levels were analyzed by western blotting. Expression levels are relative to GAPDH. ${ }^{*} \mathrm{P}<0.05$ vs. normal control; ${ }^{\#} \mathrm{P}<0.05$ vs. DACT1.

Western blot analysis. Lung tissues from patients were ground in liquid nitrogen and $100 \mathrm{mg}$ of tissue was used for RNA extraction. A total of $1 \times 10^{6}$ RAW264.7 cells were collected at $48 \mathrm{~h}$ after transfection. Pre-cooled radioimmunoprecipitation assay lysis buffer including phenylmethylsulfonyl fluoride (Thermo Fisher Scientific, Inc.) was added to the tissues and cells and incubated on ice for $5 \mathrm{~min}$. The supernatant was collected by centrifugation at $12,000 \mathrm{x} \mathrm{g}$ for $10 \mathrm{~min}$ at $4^{\circ} \mathrm{C}$. Nuclear $\beta$-catenin protein was extracted using a nucleus protein extract kit (cat. no. KGBSP002; Jiangsu KeyGen Biotech Co., Ltd., Nanjing, China), according to the manufacturer's instructions. Protein was quantified using the BCA method. A total of $5 \mu \mathrm{l}$ protein/well was separated by $10 \%$ SDS-PAGE and the proteins were transferred onto polyvinylidene fluoride membranes. Following blocking with 5\% non-fat milk for $1 \mathrm{~h}$ at room temperature, expression of DACT1 and $\beta$-catenin was detected with the following antibodies at $4^{\circ} \mathrm{C}$ overnight: Rabbit anti-DACT1 (1:1,000; cat. no. ab47473), rabbit anti- $\beta$-catenin (1:1,000; cat. no. ab16502) (both from Abcam, Cambridge, MA, USA), rabbit anti-mouse (cat. no. AH433; 1:3,000) and mouse anti-GAPDH (cat. no. A2016; 1:5,000) (both from Beyotime Institute of Biotechology, Beijing, China). Following washing with PBST for three times (15 min each time), the membrane was incubated with goat anti-rabbit IgG H\&L (HRP) (cat. no. ab6721; 1:5,000) or rabbit anti-mouse IgG H\&L (HRP) (cat. no. ab6728; Abcam, Cambridge, UK) at room temperature for $1 \mathrm{~h}$. Finally, the membrane was developed using enhanced chemiluminescence reagents (Beyotime Institute of Biotechnology). Western blot images were analyzed using Image Lab software 5.2.1 (Bio-Rad Laboratories, Inc., Hercules, CA, USA). Each experiment was repeated for three times and the expression levels of DACT1 and $\beta$-catenin were normalized against GAPDH.

Statistical analysis. Data were expressed as the mean \pm standard deviation. Statistical significance was evaluated using the paired t-test on SPSS version 16.0 software (SPSS, Inc., Chicago, IL, USA). $\mathrm{P}<0.05$ was considered to indicate a statistically significant difference.

\section{Results}

Expression of DACT in children with asthma. To evaluate the expression of DACT genes in children with asthma, DACT
mRNA and DACT protein expression levels were analyzed using RT-qPCR and western blot analysis, respectively. As demonstrated in Fig. 1A, DACT1, DACT2 and DACT3 mRNA levels were significantly upregulated in tissues from asthmatic patients compared with normal controls $(\mathrm{P}<0.05)$. The results of the western blot analysis were consistent with this, demonstrating significant upregulation of DACT1, DACT2 and DACT3 protein expression in asthmatic patients compared with normal controls $(\mathrm{P}<0.05$; Fig. 1B). In addition, DACT1 protein expression was significantly higher than DACT2 and DACT3 expression in asthmatic patients $(\mathrm{P}<0.05$; Fig. 1B). It is understood that DACT1 is a key regulator of the Wnt signaling pathway, which is closely associated with lung airway remodeling and inflammatory responses (23). Therefore, these results suggested that DACT1 may be involved in the development of asthma in children.

Effect of DACT1 on the expression of pro-inflammatory factors in RAW264.7 cells. To further evaluate the role of DACT1 in the development of asthma in children, RAW264.7 cells were used for in vitro analysis. It was identified that DACT1 mRNA expression was significantly increased compared with DACT2 and DACT3 mRNA in RAW264.7 cells ( $\mathrm{P}<0.05$; Fig. 2A). DACT1 expression was significantly inhibited by siR-DACT1 compared to the negative control $(\mathrm{P}<0.05$; Fig. $2 \mathrm{~B})$. Suppression of DACT1 expression significantly decreased the expression of IL13, IL5 and IL6 mRNA compared with the negative control $(\mathrm{P}<0.05$; Fig. 2B). Additionally, DACT1 expression was significantly enhanced by pcDNA3.1-DACT1 and overexpression of DACT1 significantly increased the expression of IL13, IL5 and IL6 mRNA compared with the empty vector control $(\mathrm{P}<0.05$; Fig. 2C), with the largest increase in IL6 expression. No significant changes in the expression level of $\beta$-catenin mRNA were observed upon DACT1 knockout or overexpression (Fig. 2B and C). These results suggested that DACT1 may upregulate the expression of pro-inflammatory factors.

Effect of DACT1 on the expression of $\beta$-catenin protein in Raw264.7 cells. Next, the effect of DACT1 on the protein expression level of $\beta$-catenin was investigated. Western blot analysis revealed that DACT1 protein expression was markedly increased compared with DACT2 and DACT3 (Fig. 3A) 

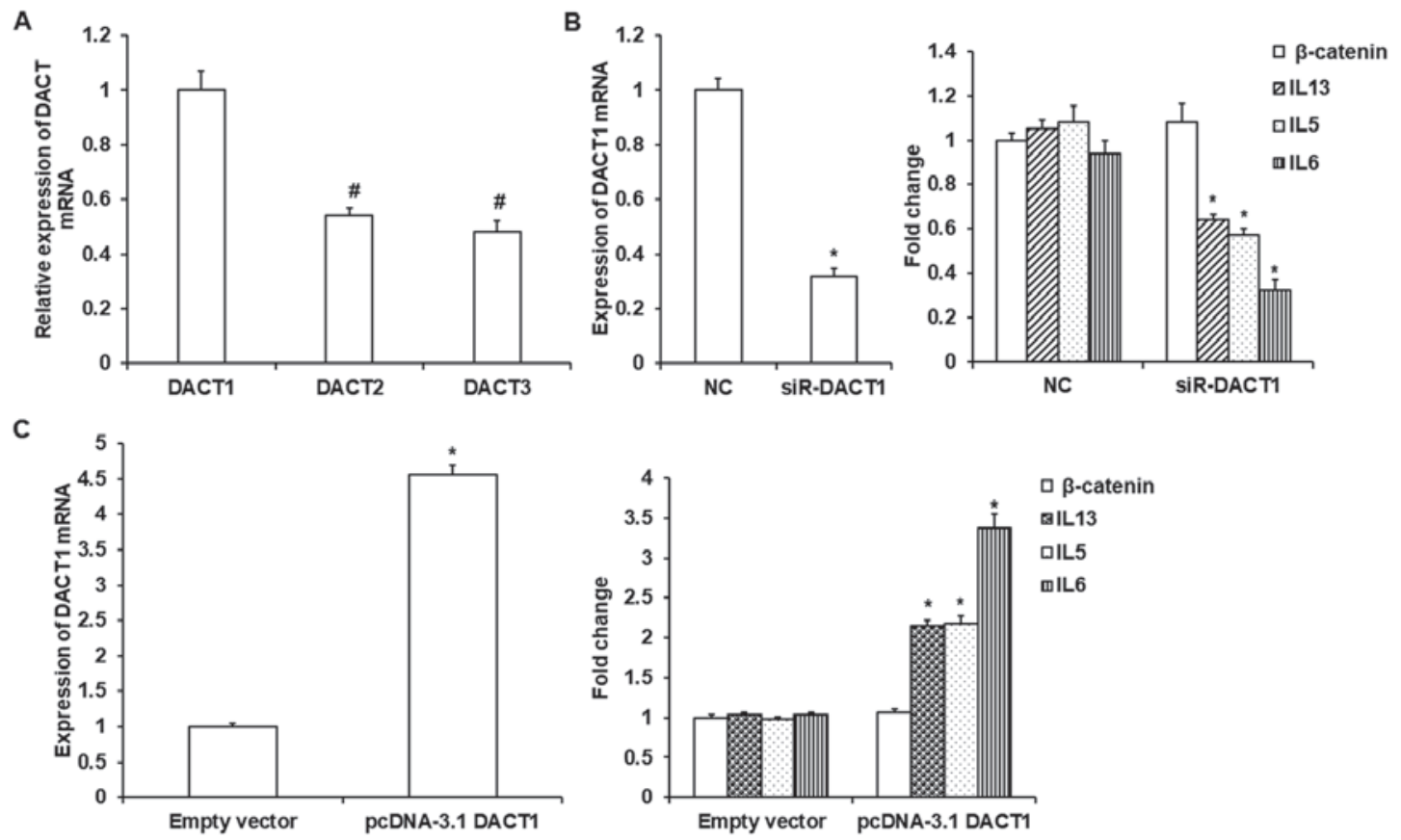

Figure 2. DACT1 promotes the expression of pro-inflammatory factors. (A) Expression of DACT1, DACT2 and DACT3 mRNA was analyzed by RT-qPCR in RAW264.7 cells. (B) RAW264.7 cells were transfected with siR-DACT1 to inhibit DACT1 expression. Expression levels of DACT1, $\beta$-catenin, IL13, IL5 and IL6 mRNA were analyzed by RT-qPCR. (C) RAW264.7 cells were transfected with pcDNA3.1-DACT1 to enhance DACT1 expression. Expression levels of DACT1, $\beta$-catenin, IL13, IL5 and IL6 mRNA were analyzed by RT-qPCR. "P $<0.05$ vs. DACT1; "P $<0.05$ vs. NC group or the empty vector. IL, interleukin; siR, small interfering RNA; NC, negative control siR; RT-qPCR, reverse transcription-quantitative polymerase chain reaction.

A
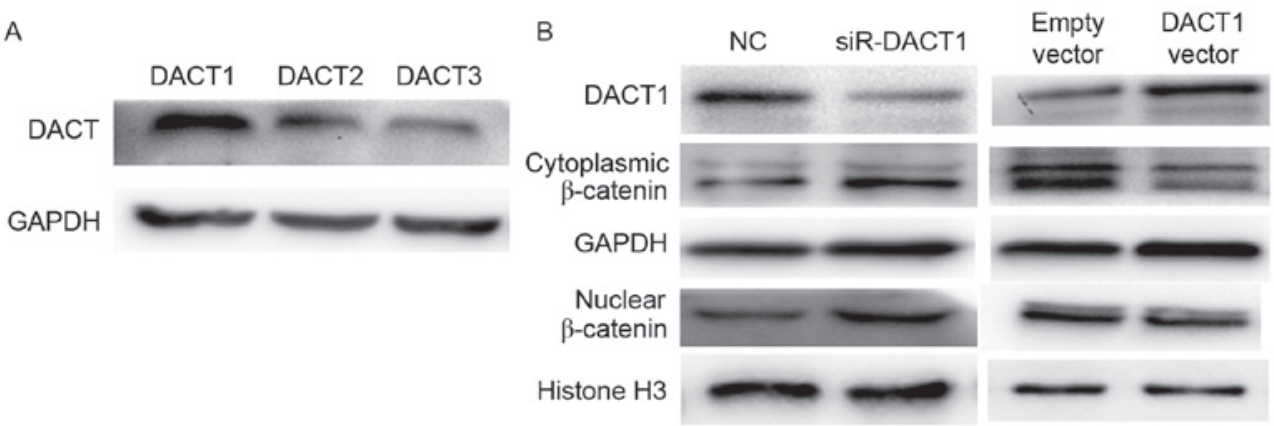

Figure 3. DACT1 inhibits the expression and nuclear translocation of $\beta$-catenin. (A) Expression levels of DACT1, DACT2 and DACT3 proteins were analyzed by western blotting in RAW264.7 cells. (B) RAW264.7 cells were transfected with siR-DACT1 (left panel) or pcDNA3.1-DACT1 (right panel) to inhibit or enhance DACT1 expression, respectively. Expression of DACT1, cytoplasmic $\beta$-catenin and nuclear $\beta$-catenin were analyzed by western blotting. siR, small interfering RNA; NC, negative control.

in RAW264.7 cells. siR-DACT1 transfection markedly downregulated DACT1 expression, while $\beta$-catenin protein expression was increased, compared with the negative control. It is understood that accumulation of $\beta$-catenin in the cytoplasm mediates the translocation of $\beta$-catenin from the cytoplasm to the nucleus, inducing the expression of multiple genes (24). Thus, nuclear $\beta$-catenin expression was evaluated and it was identified that nuclear $\beta$-catenin was upregulated when DACT1 expression was silenced compared with the negative control (Fig. 3B). Overexpression of DACT1 in RAW264.7 cells downregulated cytoplasmic and nuclear $\beta$-catenin levels compared with the empty vector (Fig. 3B). These results indicated that DACT1 may regulate the expression of pro-inflammatory factors IL13, IL5 and IL6 by modulating the expression and translocation of $\beta$-catenin.
DACT1 upregulates the expression of IL13, IL5 and IL6 by downregulating the expression and translocation of $\beta$-catenin. To further demonstrate the effect of DACT1 on the expression of $\beta$-catenin, and the subsequent regulation of pro-inflammatory factors, a rescue experiment was performed. RAW264.7 cells were transfected with pcDNA3.1-DACT1 and pcDNA3.1- $\beta$-catenin, and the expression levels of DACT1 and $\beta$-catenin were confirmed by western blot analysis (Figs. 3B and 4A). IL13, IL6 and IL5 mRNA expression levels were also evaluated using RT-qPCR. It was demonstrated that overexpression of DACT1 in RAW264.7 cells downregulated $\beta$-catenin expression compared with the empty vector (Fig. 3B) and significantly upregulated IL13, IL6 and IL5 mRNA expression compared with the negative control $(\mathrm{P}<0.05$; Fig. 4B). However, joint overexpression of $\beta$-catenin 

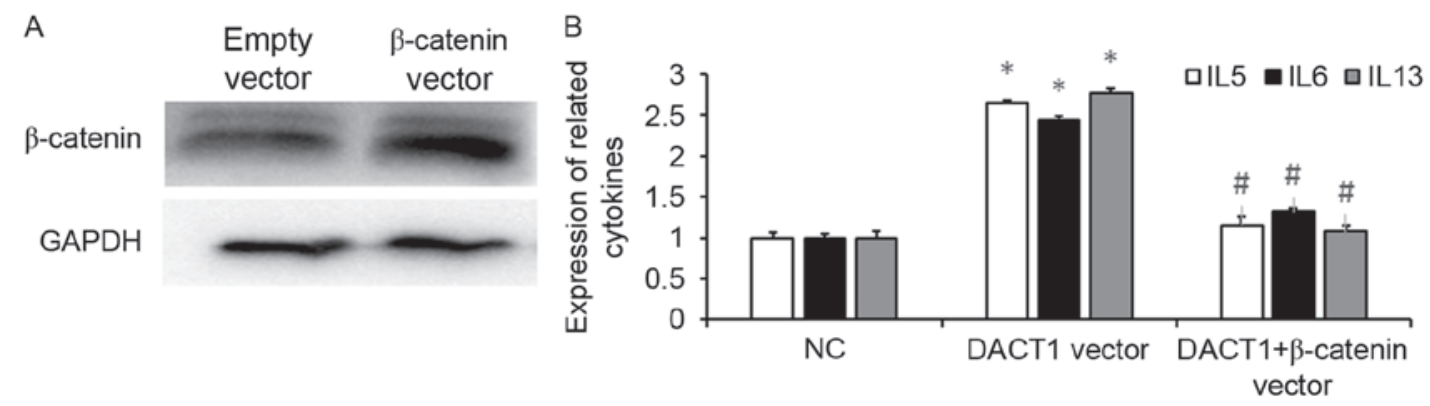

Figure 4. Rescue experiment. (A) RAW264.7 cells were transfected with pcDNA3.1- $\beta$-catenin or an empty vector, and expression of $\beta$-catenin was analyzed by western blotting. (B) RAW264.7 cells were transfected with pcDNA3.1-DACT1, empty vector or transfected with both pcDNA3.1-DACT1 and pcDNA3.1- $\beta$-catenin. Expression of IL13, IL5 and IL6 mRNA relative to GAPDH mRNA was analyzed by reverse transcription-quantitative polymerase chain reaction. ${ }^{*} \mathrm{P}<0.05$ vs. NC group; ${ }^{\mathrm{P}} \mathrm{P}<0.05$ vs. DACT1 vector. NC, negative control; IL, interleukin.
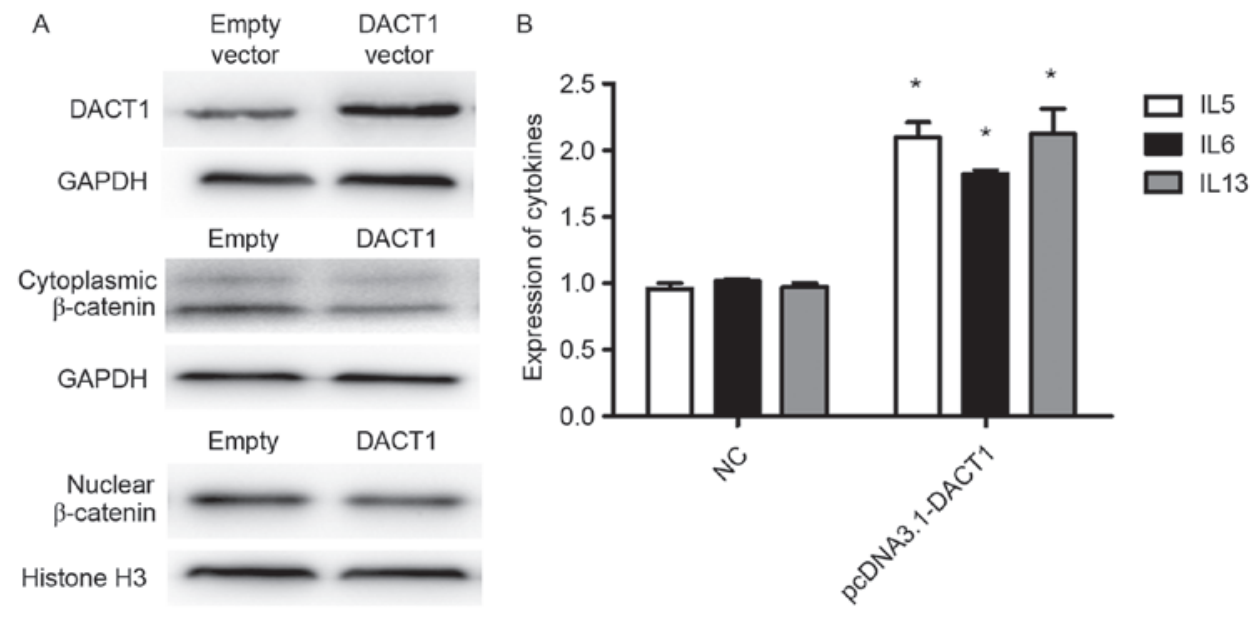

Figure 5. DACT1 suppresses the Wnt signaling pathway. RAW264.7 cells were transfected with pcDNA3.1-DACT1 and recombinant Wnt5A was added into cell culture. (A) Expression of DACT1, cytoplasmic $\beta$-catenin and nuclear $\beta$-catenin was analyzed by western blotting. (B) Expression of IL13, IL5 and IL6 mRNA relative to GAPDH mRNA was analyzed by reverse transcription-quantitative polymerase chain reaction. ${ }^{*} \mathrm{P}<0.05 \mathrm{vs}$. NC group. NC, negative control; IL, interleukin.

and DACT1 significantly downregulated expression of these three pro-inflammatory factors, compared with DACT1 overexpression alone $(\mathrm{P}<0.05$; Fig. 4B). These results further demonstrated that overexpression of $\beta$-catenin may inhibit the biological function of DACT1, and upregulation of IL13, IL 5 and IL- 6 by DACT1 was fulfilled, at least partially, via downregulation of $\beta$-catenin.

DACT1 inhibits Wnt signaling pathway activation. In order to investigate whether DACT1 could inhibit the signaling pathway downstream of Wnt, recombinant Wnt protein, Wnt5A, was added into DMEM and RAW264.7 cells were cultured for another $24 \mathrm{~h}$ to examine expression of IL13, IL6 and IL5. It was previously reported that Wnt5 is expressed at high levels in RAW264.7 cells (25). As a secretory protein, Wnt5A primarily influences adjacent cells by autocrine and paracrine actions. Therefore, Wnt5A was used to activate the Wnt signaling pathway in RAW264.7 cells. Following the addition of Wnt5A, pcDNA3.1-DACT1 transfection markedly repressed cytoplasmic $\beta$-catenin expression and the nuclear translocation of $\beta$-catenin compared with empty vector transfected cells (Fig. 5A), while expression of IL13, IL6 and IL5 mRNA was significantly increased compared with the negative control $(\mathrm{P}<0.05$; Fig. 5B). As nuclear translocation of $\beta$-catenin is an important marker of Wnt signaling pathway activation (26), these results indicated that DACT1 could inhibit the Wnt signaling pathway. Therefore, DACT1 may release the inflammatory factors that were formerly repressed by Wnt, thus promoting local inflammation.

\section{Discussion}

In the present study, it was identified that DACT1 gene expression was upregulated in lung biopsies from children with asthma compared with healthy controls. Further evaluation at the molecular level revealed that DACT1 was able to inhibit the expression of $\beta$-catenin and its translocation into the nucleus in RAW264.7 cells. DACT1 also inactivated the Wnt signaling pathway, likely through inhibiting the nuclear translocation of $\beta$-catenin, leading to the upregulation of inflammatory cytokines IL13, IL5 and IL6. This may be related to the development of asthma in children.

Previous evidence indicates that inflammation has an important role in the development of asthma, and various cytokines secreted by inflammatory cells regulate airway 
hyperresponsiveness and remodeling $(27,28)$. For instance, IL13 secreted by Th2 cells may directly stimulate the proliferation of lung fibroblasts (29). IL13 may also induce vascular endothelial growth factor secretion by bronchial smooth muscle cells, increasing airway remodeling (30,31). IL4 may regulate signal transducer and activator of transcription 6 signaling pathways to participate in the airway inflammation process (32). DACT1 is a negative regulator of the Wnt signaling pathway, which may exert a very important function. ACT1 is involved in the pathophysiology of cell proliferation, apoptosis, differentiation and migration through negatively regulating the Wnt signaling pathway (33). A study by Ma et al (34) demonstrated that DACT1 promoted the degradation of dishevelled 2 protein. The Wnt signaling pathway is also correlated with airway inflammation. A study by Guo et al (35) indicated that the $\mathrm{Wnt} / \beta$-catenin singling pathway suppressed airway inflammatory responses by regulating the peroxisome proliferator-activated receptor $\delta / \mathrm{p} 38$ pathway. A study by Dong et al (36) demonstrated that the Wnt signaling pathway was able to regulate the expression of IL1 $\beta$, IL10 and IL6 in hepatic stellate cells, thus increasing tissue inflammation. $\beta$-catenin was identified as a key effector in the Wnt signaling pathway, and its biological function could be regulated by control of its expression level or nuclear translocation (37). A study by Yuan et al (15) demonstrated that DACT1 was a positive regulator of $\beta$-catenin; however, the correlation of DACT1 and $\beta$-catenin in asthma remains unclear. The present results indicated that upregulation of DACT1 expression could inhibit the expression and nuclear translocation of $\beta$-catenin in macrophage cell line, RAW264.7, as well as upregulate the expression of IL13, IL5 and IL6 mRNA. In contrast, inhibition of $\beta$-catenin expression in this system significantly suppressed this biological function of DACT1. These results indicated that DACT1 may promote airway inflammation in patients with asthma, thus promoting the development of asthma by regulation of the expression and nuclear translocation of $\beta$-catenin.

In conclusion, the present study indicated that DACT1 expression is upregulated in tissues from asthmatic patients. In vitro experiments demonstrated that silencing the expression of DACT1 promoted the expression and nuclear translocation of $\beta$-catenin in RAW264.7 cells, and suppressed the expression of IL13, IL5 and IL6. These findings indicate that DACT1 may be a potential target for alleviating the airway inflammatory response in asthma.

\section{Acknowledgements}

The present study was supported by the Jining Chinese Medicine Science and Technology Development Plan (grant no. Jiningzy-079-009). The authors would like to thank Dr. Bosong Song and Director Dong Bai for critical discussion.

\section{References}

1. Stevens W, Buchheit K and Cahill KN: Aspirin-exacerbated diseases: Advances in asthma with nasal polyposis, urticaria, angioedema and anaphylaxis. Curr Allergy Asthma Rep 15: 69, 2015.

2. Nabih ES, Kamel HF and Kamel TB: Association between CD14 polymorphism (-1145G/A) and childhood bronchial asthma. Biochem Genet 54: 50-60, 2016.
3. Dissanayake S, Jain M, Grothe B, Mciver T and Papi A: An evaluation of comparative treatment effects with high and low dose fluticasone propionate/formoterol combination in asthma. Pulm Pharmacol Ther 35: 19-27, 2015.

4. Pinto S, McCrone S and Shapiro AL: Perceptions of asthma quality of life in children and parent dyads in two rural counties in west virginia. J Sch Nurs 32: 267-72, 2016.

5. Niu C, Liu N, Liu J, Zhang M, Ying L, Wang L, Tian D, Dai J, Luo Z, Liu E, et al: Vitamin A maintains the airway epithelium in a murine model of asthma by suppressing glucocorticoid-induced leucine zipper. Clin Exp Allergy 46: 848-860, 2016.

6. Cazzola M, Coppola A, Rogliani P and Matera MG: Novel glucocorticoid receptor agonists in the treatment of asthma. Expert Opin Investig Drugs 24: 1473-1482, 2015.

7. Sears MR: Predicting asthma outcomes. J Allergy Clin Immunol 136: 829-836, 2015.

8. Chiappori A, De Ferrari L, Folli C, Mauri P, Riccio MA and Canonica WG: Biomarkers and severe asthma: A critical appraisal. Clin Mol Allergy 13: 20, 2015.

9. Matusovsky OS, Kachmar L, Ijpma G, Bates G, Zitouni N, Benedetti A, Lavoie JP and Lauzon AM: Peripheral airway smooth muscle but not the trachealis is hypercontractile in an equine model of asthma. Am J Respir Cell Mol Biol 54: 718-727, 2016.

10. Wu Q, Tang Y, Hu X, Wang Q, Lei W, Zhou L and Huang J: Regulation of Th1/Th2 balance through OX40/OX40L signalling by glycyrrhizic acid in a murine model of asthma. Respirology 21 : 102-111, 2016.

11. Ma B, Liu B, Cao W, Gao C, Qi Z, Ning Y and Chen YG: The Wnt signaling antagonist dapper1 accelerates Dishevelled2 degradation via promoting its ubiquitination and aggregate-induced autophagy. J Biol Chem 290: 12346-12354, 2015.

12. Yin X, Xiang T, Li L, Su X, Shu X, Luo X, Huang J, Yuan Y, Peng W, Oberst M, et al: DACT1, an antagonist to Wnt $/ \beta$-catenin signaling, suppresses tumor cell growth and is frequently silenced in breast cancer. Breast Cancer Res 15: R23, 2013.

13. Deng J, Liang H, Zhang R, Ying G, Xie X, Yu J, Fan D and Hao X: Methylated CpG site count of dapper homolog 1 (DACT1) promoter prediction the poor survival of gastric cancer. Am J Cancer Res 4: 518-527, 2014.

14. Yin X, Xiang T, Li L, Su X, Shu X, Luo X, Huang J, Yuan Y, Peng W, Oberst M, et al: DACT1, an antagonist to Wnt/ $\beta$-catenin signaling, suppresses tumor cell growth and is frequently silenced in breast cancer. Breast Cancer Res 15: R23, 2013.

15. Yuan G, Wang C, Ma C, Chen N, Tian Q, Zhang T and Fu W: Oncogenic function of DACT1 in colon cancer through the regulation of $\beta$-catenin. PLoS One 7: e34004, 2012.

16. Arguello A and Cheyette BN: Dapper antagonist of catenin-1 (Dact1) contributes to dendrite arborization in forebrain cortical interneurons. Commun Integr Biol 6: e26656, 2013.

17. Yang X, Fisher DA and Cheyette BN: SEC14 and spectrin domains 1 (Sestd1), dishevelled 2 (Dvl2) and dapper antagonist of catenin-1 (Dact1) co-regulate the Wnt/planar cell polarity (PCP) pathway during mammalian development. Commun Integr Biol 6: e26834, 2013.

18. Guo Y, Mishra A, Howland E, Zhao C, Shukla D, Weng T and Liu L: Platelet-derived Wnt antagonist Dickkopf-1 is implicated in ICAM-1/VCAM-1-mediated neutrophilic acute lung inflammation. Blood 126: 2220-2229, 2015.

19. Kumawat K, Koopmans T and Gosens R: $\beta$-catenin as a regulator and therapeutic target for asthmatic airway remodeling. Expert Opin Ther Targets 18: 1023-1034, 2014.

20. Song P, Zheng JX, Liu JZ, Xu J, Wu LY, Liu C, Zhu Q and Wang Y: Effect of the Wnt $1 / \beta$-catenin signalling pathway on human embryonic pulmonary fibroblasts. Mol Med Rep 10: 1030-1036, 2014

21. Katoh M and Katoh M: Transcriptional mechanisms of WNT5A based on NF-kappaB, Hedgehog, TGFbeta, and Notch signaling cascades. Int J Mol Med 23: 763-769, 2009.

22. Livak KJ and Schmittgen TD: Analysis of relative gene expression data using real-time quantitative PCR and the 2(-Delta Delta C(T)) method. Methods 25: 402-408, 2001.

23. Yang ZQ, Zhao Y, Liu Y, Zhang JY,Zhang S, Jiang GY, Zhang PX, Yang LH, Liu D, Li QC and Wang EH: Downregulation of HDPR 1 is associated with poor prognosis and affects expression levels of p120-catenin and beta-catenin in nonsmall cell lung cancer. Mol Carcinog 49: 508-519, 2010.

24. Cai Y,Zhu S, Yang W, Pan M, Wang C and Wu W: Downregulation of $\beta$-catenin blocks fibrosis via Wnt2 signaling in human keloid fibroblasts. Tumour Biol 39: 1010428317707423, 2017. 
25. Qin L, Hu R, Zhu N, Yao HL, Lei XY, Li SX, Liao DF and Zheng XL: The novel role and underlying mechanism of Wnt5a in regulating cellular cholesterol accumulation. Clin Exp Pharmacol Physiol 41: 671-678, 2014.

26. Polley A, Sen P, Sengupta A and Chakraborty S: $\beta$-catenin stabilization promotes proliferation and increase in cardiomyocyte number in chick embryonic epicardial explant culture. In Vitro Cell Dev Biol Anim: Aug 25, 2017 (Epub ahead of print).

27. Taniguchi A, Miyahara N, Waseda K, Kurimoto E, Fujii U, Tanimoto $Y$, Kataoka M, Yamamoto Y, Gelfand EW, Yamamoto $\mathrm{H}$, et al: Contrasting roles for the receptor for advanced glycation end-products on structural cells in allergic airway inflammation vs. airway hyperresponsiveness. Am J Physiol Lung Cell Mol Physiol 309: L789-L800, 2015.

28. Hoppenot D, Malakauskas K, Lavinskiene S and Sakalauskas R p-STAT6, PU.1, and NF- $\kappa$ B are involved in allergen-induced late-phase airway inflammation in asthma patients. BMC Pulm Med 15: 122, 2015.

29. Li WL, Xiong LX, Shi XY, Xiao L, Qi GY and Meng C: IKK $\beta / N F \kappa B p 65$ activated by interleukin-13 targets the autophagy-related genes LC3B and beclin 1 in fibroblasts co-cultured with breast cancer cells. Exp Ther Med 11: 1259-1264, 2016.

30. Nakajima M, Honda T, Miyauchi S and Yamazaki K: Th2 cytokines efficiently stimulate periostin production in gingival fibroblasts but periostin does not induce an inflammatory response in gingival epithelial cells. Arch Oral Biol 59: 93-101, 2014.
31. Corren J: Role of interleukin-13 in asthma. Curr Allergy Asthma Rep 13: 415-420, 2013.

32. Chiba Y, Todoroki M and Misawa M: Interleukin-4 upregulates RhoA protein via an activation of STAT6 in cultured human bronchial smooth muscle cells. Pharmacol Res 61: 188-192, 2010.

33. Jardim DP, Poço PCE and Campos AH: Dact1, a Wnt-pathway inhibitor, mediates human mesangial cell TGF- $\beta 1$-induced apoptosis. J Cell Physiol 232: 2104-2111, 2017.

34. Ma B, Liu B, Cao W, Gao C, Qi Z, Ning Y and Chen YG: The Wnt signaling antagonist dapperl accelerates Dishevelled2 degradation via promoting its ubiquitination and aggregate-induced autophagy. J Biol Chem 290: 12346-12354, 2015.

35. Guo L, Wang T, Wu Y, Yuan Z, Dong J, Li X, An J, Liao Z, Zhang $\mathrm{X}, \mathrm{Xu} \mathrm{D}$ and Wen FQ: WNT/ $\beta$-catenin signaling regulates cigarette smoke-induced airway inflammation via the PPARס/p38 pathway. Lab Invest 96: 218-229, 2016.

36. Dong S, Wu C, Hu J, Wang Q, Chen S, Wang Z and Xiong W: Wnt5a promotes cytokines production and cell proliferation in human hepatic stellate cells independent of canonical Wnt pathway. Clin Lab 61: 537-547, 2015.

37. Tao H, Chen F, Liu H, Hu Y, Wang Y and Li H: Wnt/ $\beta$-catenin signaling pathway activation reverses gemcitabine resistance by attenuating Beclin1-mediated autophagy in the MG63 human osteosarcoma cell line. Mol Med Rep 16: 1701-1706, 2017. 